
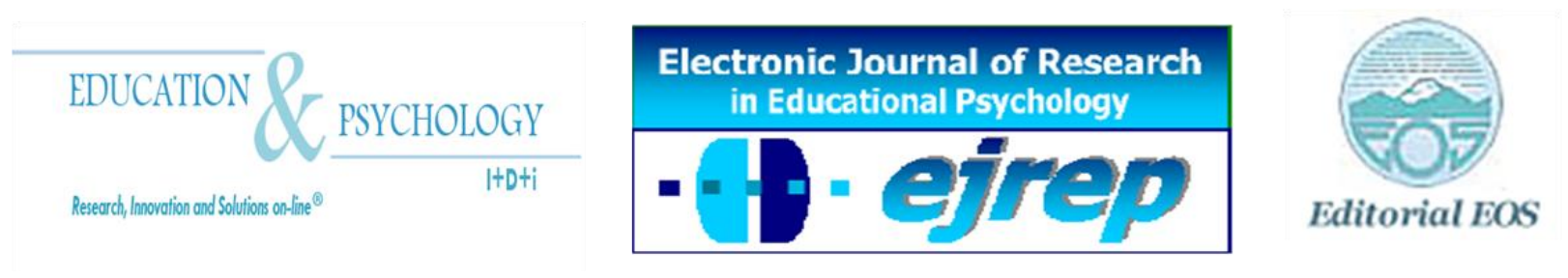

\title{
Estrategias educativas para orientar las necesidades educativas de los estudiantes con Síndrome de Asperger en aulas ordinarias
}

\section{Leire Darretxe Urrutxi ${ }^{1}$ y Lucy Sepúlveda Velásquez ${ }^{2}$}

\author{
${ }^{1}$ Didáctica y Organización Escolar, Universidad del País Vasco, Bilbao. \\ ${ }^{2}$ Universidad Academia de Humanismo Cristiano, Santiago.
}

\section{España / Chile}

Correspondencia: Leire Darretxe Urrutxi. Departamento de Didáctica y Organización Escolar. Universidad del País Vasco. Ramón y Cajal 72, C.P. 48014 Bilbao. E-mail: leire.darreche@ehu.es

() Education \& Psychology I+D+i and Editorial EOS (Spain) 


\section{Resumen}

En el presente artículo se realiza una descripción del Síndrome Asperger (SA), trastorno que se enmarca dentro del espectro autista. Con el fin de que el lector o lectora comprenda mejor las características de dicho trastorno, se describen las dificultades básicas que manifiestan dichas personas referidas, principalmente, a la interacción social, comunicación e imaginación, aportándose algunos casos puntuales que dan una visión más concreta del tema. Considerando las principales teorías explicativas del síndrome: "teoría de la mente", "la coherencia central" y "las funciones ejecutivas", se detallan varias implicaciones educativas y estrategias de intervención que, en contraste con nuestra propia experiencia, resultan útiles en las aulas para responder a las necesidades de estos estudiantes en entornos ordinarios. Abordar las concepciones del SA desde la dimensión afectiva, de relación y cognición, posibilita una mejor comprensión e intervención educativa hacia estas personas, mejorando el desarrollo de sus habilidades sociales, de comunicación, de lenguaje, de autorregulación y de autodeterminación, y que en síntesis, equivale a potenciar su calidad de vida en el contexto educativo de los centros y aulas inclusivas.

Palabras Clave: Síndrome de Asperger, Necesidades Educativas Especiales, Inclusión, Teorías psicológicas, Intervenciones educativas. 


\title{
Educational strategies to guide the educational needs of students with Asperger Syndrome in the mainstream classroom
}

\begin{abstract}
The following article describes the Asperger's syndrome (AS), an autism spectrum disorder. In order that the reader may better comprehend the characteristics of this disorder, the main difficulties that these people have to face, especially with regard to social interaction, communication and imagination, are explained. In addition, some specific examples illustrate this and give a better overview of the topic.

Several educative implications and intervention strategies are described in detail based on the main explanatory theories of this syndrome: "theory of the mind", "central coherence" and "executive functions". Contrary to our own experiences, these strategies end up being useful in class for satisfying the needs of such students in ordinary environments. Dealing with AS conceptions from an affective dimension, one of relationship and cognition, makes a better understanding and improved educative interventions towards these people possible, which boosts their developing their social, communicative, language, self-regulation and selfdetermination capabilities. In summary, this results in promoting their living quality in the context of inclusive classrooms and institutions.
\end{abstract}

Keywords: Asperger Syndrome, Special Needs, Inclusion, Psychological Theories, Educational Interventions. 


\section{Introducción}

¿Por qué hemos elegido el síndrome de Asperger ${ }^{1}$ ? ¿Qué hecho circunstancial nos impulsó hacia la elaboración de estas pautas orientativas relacionadas con la inclusión educativa del alumnado con este trastorno? Quienes hemos sido testigos de la escolarización de estos estudiantes con síndrome Asperger o Autismo de Alto Funcionamiento (Klin y Volkmar, 1996; Munro, 1999 y Moreno y O’Neal, 2003) ${ }^{2}$ en el aula regular durante estos últimos años, creemos que es de rigor abordar esta realidad educativa con el fin de favorecer la inclusión ya que: ¿Cuántas de estas personas no diagnosticadas y con estas características han pasado por los centros ordinarios? en tanto que el docente, inexperto en el tema, tendía a considerarlos, por una parte, como estudiantes "normales" por su aspecto físico, pero con intereses insólitos en cosas que los demás, ni el propio profesor o profesora dominaban. Es decir, en ciertas situaciones estos estudiantes "deslumbraban"; sin embargo, en otras destacaban por su ingenuidad, tono de voz, rabietas imprecisas y singularidades cambiantes de humor que descentraban originando cierto caos en el aula. Por consiguiente, el objetivo de este artículo es el de proponer orientaciones educativas para favorecer la inclusión de los estudiantes con Síndrome de Asperger en el aula ordinaria. Ser testigos privilegiados de intervenciones educativas, nos ha posibilitado vislumbrar ciertos horizontes y profundizar en el tema, de ahí que las contingencias presentadas en este artículo nos permiten responder a las inquietudes docentes de manera precisa, con el objeto de orientarlos y perfilar ciertos patrones de actuación para que sirvan de guía en su labor inclusiva hacia el alumnado con estas características.

En cuanto a los instrumentos para el diagnóstico del síndrome que más se utilizan son, por una parte, el "Manual Diagnóstico y Estadístico de Trastornos Mentales" (DSM-IV) publicado por la Asociación Americana de Psiquiatría en 1994; y por otra parte, la “Clasificación Internacional de Enfermedades" (CIE), emitida por la Organización Mundial de la Salud en 1992. Actualmente el diagnóstico más reciente, a nivel internacional,

\footnotetext{
${ }^{1}$ El Síndrome de Asperger es un trastorno del desarrollo y por esa razón en el artículo se alude a síndrome o a trastorno indistintamente.

${ }^{2}$ La evidencia de esta argumentación relativa al uso de las dos acepciones: Síndrome de Asperger o Autismo de Alto Funcionamiento, las encontramos en el DSM-IV-TR (2002), aspectos que también desarrollan y resumen, indistintamente, Klin y Volkmar (1996); Munro (1999) y Moreno y O’Neal (2003), entre otros. Ellos aportan elementos característicos y congruentes de ambas identidades (En Sepúlveda 2009, p. 139).
} 
corresponde al expresado en el "DSM-IV-TR (2002)", y se prevé que en la nueva edición del "DSM-V", el único diagnóstico posible sea el trastorno del espectro de autismo, eliminando, de este modo, el SA como categoría independiente (Ghaziuddin, 2010). No obstante, hasta que no se publique una nueva edición se debe continuar con lo que actualmente conocemos "DSM-IV-TR (2002)". La descripción referida al SA en dicho Manual, es descrito como un Trastorno Generalizado del Desarrollo (TGD) que afecta a un extenso rango de habilidades, caracterizado por la dificultad en las siguientes áreas: a) la interacción social, b) la comunicación [verbal y no verbal] y c) la imaginación, aspectos recogidos por la mayoría de la bibliografía revisada y conocidos como la triada de síntomas observables (Alberta Learning, 2003; Winter, 2005; Bogdashina, 2005; Pittman, 2007). Señalamos que ambos sistemas de diagnósticos están basados en las dificultades de dicha triada.

\section{Principales particularidades del Síndrome de Asperger}

Las personas con SA suelen presentar ciertas características que resultan convenientes conocer para dar una mejor respuesta a sus necesidades. Concretamente, algunas de esas características, que suelen reflejarse también en la escuela y en las aulas son, según Myles et. al. (2005, p.7), las siguientes:

\begin{tabular}{|l|l|}
\hline Intereses limitados hacia tópicos específicos & Baja tolerancia a la frustración \\
\hline Dificultad con los cambios en la rutina & Pobres estrategias de imitación \\
\hline Incapacidad para hacer amigos y amigas & Rango de intereses restringido \\
\hline Dificultad en las conversaciones recíprocas & Problemas de motricidad fina \\
\hline Discurso pedante & Pobre concentración \\
\hline $\begin{array}{l}\text { Socialmente personas ingenuas y de pensamiento } \\
\text { literal }\end{array}$ & Dificultades académicas \\
\hline Tendencia a ser vulnerables & Vulnerabilidad emocional \\
\hline Dificultad para aprender en grupos grandes & Habilidades de organización pobres \\
\hline Dificultades con conceptos abstractos & Apariencia "normal" para otras personas \\
\hline Pobres habilidades de resolución de problemas & Torpeza motora \\
\hline $\begin{array}{l}\text { Vocabulario por lo general amplio. Pobre } \\
\text { comprensión }\end{array}$ & $\begin{array}{l}\text { Aspectos sensoriales (problemas al } \\
\text { procesar la información) }\end{array}$ \\
\hline
\end{tabular}

Figura 1. Algunos rasgos característicos del síndrome

A pesar de que todas las personas con SA tengan ciertas particularidades comunes, las características individuales explican la variedad de casos existentes. Para ello y según los 
estudios realizados por Cumine, Leach y Stevenson (2000), presentaremos, a modo de ejemplo, tres casos significativos ${ }^{3}$, que describen la individualidad del síndrome:

- George (seis años): Lo definen como un niño pasivo que no tiene interés en otros niños y niñas. Su preferencia es completar la misma forma de tablero una y otra vez. Muchos de sus iguales han dejado de intentar interactuar con él y otros le tratan cruelmente, por ejemplo, echándole una pelota para que la vaya a recoger como si se tratara de un perro. Tiene un lenguaje extraño, sobre todo al responder a preguntas y suele tener expresiones faciales no acordes con el contexto. Leyendo y deletreando es muy ágil, pero en la comprensión muestra dificultades. En ocasiones camina de puntillas y agita sus manos. Le gusta tener un trozo pequeño de cuerda para retorcerla.

- Michael (ocho años): Busca involucrar a otras personas en el juego, pero en vez de pedírselo les agarra físicamente. A sus iguales les extraña el lenguaje que utiliza ya que es "pasado de moda". No entiende las consecuencias de sus actos en los sentimientos de los demás. Tiene excelentes habilidades para construir juguetes, pero nunca los comparte con nadie. Muestra interés por el cableado eléctrico, sin tener conciencia de los peligros que ocasiona. El sonido del viento le hace gritar.

- Jeff (14 años): A la hora de comida se muestra muy ansioso por el ruido y el bullicio que se crea en el comedor. No le gusta ser observado mientras come. En su casa, si hay visitas, come en su habitación. En grupo observa las actividades que se realizan, pero siempre parece estar fuera de lugar. Habla sin parar de agricultura, si consigue a alguien que le escuche. En Educación Física nadie quiere formar con él equipo. Tiene una coordinación pobre.

\footnotetext{
${ }^{3}$ Otros ejemplos los encontramos en Wall (2007).
} 


\section{Estrategias educativas a partir de las principales teorías}

En este apartado se explican, brevemente, algunas de las principales teorías psicológicas que intentan comprender a las personas con autismo debido a las dificultades que muestran en estos ámbitos, nos referimos concretamente a: "la teoría de la mente", "la coherencia central" y "las funciones ejecutivas". Comprender estas teorías resulta relevante ya que permite entender las implicaciones educativas que pueden resultar útiles y beneficiosas con estos individuos. Para este caso señalamos a Stichter, et al. (2010), quienes manifiestan la eficacia de las intervenciones de la competencia social diseñada para personas con SA. Por consiguiente, nuestra intención es que, partiendo de estas teorías, aportemos ideas para una respuesta educativa idónea.

\section{La teoría de la mente}

Premack y Woodruff (1978) introdujeron el término de "teoría de la mente" para describir la capacidad de las personas para atribuir estados mentales a uno mismo y a los demás y para predecir el comportamiento de los demás. Investigaciones posteriores como la de Leslie (1987), Frith (1989), Baron-Cohen (2000), entre otros y otras, han demostrado que las personas con autismo (incluidas las personas con SA) muestran dificultades en la teoría de la mente.

Un estudio realizado por Baron-Cohen, Leslie y Frith (1985) resulta muy significativo al respecto ya que presentando, a través del experimento previo realizado por Wimmer y Perner (1983, en Shaffer, 2000, p. 227) la historia de Sally y Anne, probaron cómo las personas con autismo tienen dificultades en la teoría de la mente. Posteriormente, Cumine, Leach y Stevenson (2000) concluyeron que las personas con SA tienen una serie de dificultades en diferentes áreas, según las cuales, a nivel educativo, se pueden establecer unos principios de intervención consistentes en:

\begin{tabular}{|l|l|}
\hline \multicolumn{2}{|c|}{ DIFICULTADES Y PRINCIPIOS DE INTERVENCIÓN A NIVEL ESCOLAR } \\
\hline \multicolumn{1}{|c|}{ DIFICULTADES EN } & \multicolumn{1}{|c|}{ PRINCIPIOS DE INTERVENCIÓN } \\
\hline $\begin{array}{l}\text { Predecir el comportamiento de otras personas, } \\
\text { prefiriendo actividades en las que no dependen } \\
\text { o no requieren de la ayuda de otras personas. }\end{array}$ & $\begin{array}{l}\text { Aprender habilidades sociales para el aula o el } \\
\text { patio del colegio puede resultar muy estresante } \\
\text { para los estudiantes con SA. En este sentido se } \\
\text { debe saber que el mantener las tareas }\end{array}$ \\
\hline
\end{tabular}




\begin{tabular}{|c|c|}
\hline & $\begin{array}{l}\text { académicas es un alivio para contrarrestar el } \\
\text { estrés de la interacción social. }\end{array}$ \\
\hline $\begin{array}{l}\text { Leer las intenciones de las otras personas y } \\
\text { entender los motivos de sus comportamientos. }\end{array}$ & $\begin{array}{l}\text { Se deberán enseñar las habilidades básicas } \\
\text { sociales de escuchar, no interrumpir, realizar } \\
\text { pausas para permitir a otras personas hablar, } \\
\text { esperar en fila, trabajar en pequeños grupos... }\end{array}$ \\
\hline Explicar la propia conducta. & $\begin{array}{l}\text { Necesitan ayuda para reconocer el efecto de sus } \\
\text { acciones en los demás y modificar su } \\
\text { comportamiento en consecuencia. }\end{array}$ \\
\hline $\begin{array}{l}\text { Entender las emociones (tanto las propias como } \\
\text { la de los demás), derivando en una falta de } \\
\text { empatía. Entender que el comportamiento } \\
\text { afecta en lo que piensen o sientan los demás, lo } \\
\text { que les lleva a la falta de consciencia y de } \\
\text { motivación para pedir ayuda. }\end{array}$ & $\begin{array}{l}\text { Debemos ser cautos a la hora de suponer que el } \\
\text { nivel de comunicación es lo que representa su } \\
\text { lenguaje. }\end{array}$ \\
\hline $\begin{array}{l}\text { Entender que el comportamiento afecta en lo } \\
\text { que piensen o sientan los demás, lo que les lleva } \\
\text { a la falta de consciencia y de motivación para } \\
\text { pedir ayuda. }\end{array}$ & $\begin{array}{l}\text { Ser explícitos al dar instrucciones, sin asumir } \\
\text { que el contexto aclara el significado. }\end{array}$ \\
\hline $\begin{array}{l}\text { Tomar en cuenta lo que los demás saben o } \\
\text { deberían saber, soliendo usar un lenguaje } \\
\text { pedante e incomprensible. }\end{array}$ & $\begin{array}{l}\text { Utilizar estrategias para desarrollar el } \\
\text { autoconcepto, la autoestima... }\end{array}$ \\
\hline $\begin{array}{l}\text { Reaccionar a los intereses de la persona que } \\
\text { escucha cuando se está hablando. Anticipar lo } \\
\text { que puedan pensar los demás de las propias } \\
\text { acciones. }\end{array}$ & $\begin{array}{l}\text { Enseñar a identificar las emociones "in situ", } \\
\text { llamando la atención sobre las expresiones de } \\
\text { emoción de los demás. }\end{array}$ \\
\hline $\begin{array}{l}\text { Anticipar lo que puedan pensar los demás de } \\
\text { las propias acciones. }\end{array}$ & $\begin{array}{l}\text { Garantizar que la persona está prestando } \\
\text { atención a la actividad que se esté realizando. }\end{array}$ \\
\hline Engañar o entender el engaño. & $\begin{array}{l}\text { Alertarle de su rol en las tareas, las situaciones y } \\
\text { los eventos. }\end{array}$ \\
\hline $\begin{array}{l}\text { Compartir la atención con los demás, } \\
\text { derivando en una referencia idiosincrásica. }\end{array}$ & $\begin{array}{l}\text { Llamar la atención para el uso de gestos, } \\
\text { expresiones faciales, la dirección de la mirada, } \\
\text { la proximidad en la interacción social... }\end{array}$ \\
\hline $\begin{array}{l}\text { Comprender la interacción social, dando lugar } \\
\text { a las dificultades en la toma de turnos, uso } \\
\text { inadecuado del contacto visual... }\end{array}$ & $\begin{array}{l}\text { Tener cuidado al asumir que el estudiante con } \\
\text { SA ha entendido las intenciones de nuestra } \\
\text { conducta. }\end{array}$ \\
\hline Diferenciar la realidad de la ficción. & Ayudarle a diferenciar la ficción de la realidad. \\
\hline
\end{tabular}

Figura 2. Dificultades y principios de intervención a nivel escolar

\section{La coherencia central}

Uta Frith (1989) señaló que no todos los aspectos del funcionamiento en el autismo eran explicados por la "teoría de la mente", como por ejemplo, la insistencia en mantener la rutina, preocupaciones obsesivas, la atención por los detalles, etc. La misma autora describió en 1989 la "coherencia central", como la tendencia a reunir diferentes datos con el fin de obtener la mayor información posible del contexto, es decir, tendencia a dar sentido a las situaciones y eventos según la realidad, aspecto en el cual las personas con SA muestran dificultad. 
A continuación señalamos las principales implicaciones del déficit de la coherencia central y los principios de intervención, según Cumine, Leach y Stevenson (2000):

\begin{tabular}{|l|l|}
\hline \multicolumn{2}{|c|}{ DIFICULTADES Y PRINCIPIOS DE INTERVENCIÓN A NIVEL ESCOLAR } \\
\hline \multicolumn{1}{|c|}{ DIFICULTADES EN } & \multicolumn{1}{c|}{ PRINCIPIOS DE INTERVENCIÓN } \\
\hline Focos de atención idiosincrásicos. & $\begin{array}{l}\text { Marcar un comienzo y un final claro en las } \\
\text { tareas, por ejemplo, usar una lista de pasos } \\
\text { hasta que la tarea esté completada pudiendo } \\
\text { usar pictogramas, diagramas... }\end{array}$ \\
\hline Imposición de la propia perspectiva. & $\begin{array}{l}\text { Considerar alguna imagen de la tarea final } \\
\text { para que los estudiantes con SA sepan qué es } \\
\text { lo que se espera. }\end{array}$ \\
\hline Preferencia por los conocimientos. & $\begin{array}{l}\text { Evitar la ambigüedad, usando pistas visuales } \\
\text { que resalten el significado. }\end{array}$ \\
\hline Falta de atención sobre las tareas nuevas. & Enseñarles a tomar decisiones. \\
\hline Dificultad en elegir y priorizar. & Enseñarles a tomar decisiones. \\
\hline $\begin{array}{l}\text { Dificultad en la organización de uno mismo, } \\
\text { de los materiales, experiencias... }\end{array}$ & $\begin{array}{l}\text { Crear oportunidades para que puedan } \\
\text { generalizar el conocimiento y las } \\
\text { competencias. }\end{array}$ \\
\hline $\begin{array}{l}\text { Dificultad para ver las conexiones y } \\
\text { generalizar el conocimiento y las } \\
\text { competencias. }\end{array}$ & $\begin{array}{l}\text { Enseñarles historias usando secuencias con } \\
\text { pictogramas. En este sentido las historias } \\
\text { sociales de Carol Gray pueden ser útiles. }\end{array}$ \\
\hline
\end{tabular}

Figura 3. Principales implicaciones del déficit de la coherencia central y principios de intervención.

\section{Las funciones ejecutivas}

Dichas funciones hacen referencia a la capacidad de mantener una adecuada resolución de problemas para la realización de una meta en el futuro. Generalizando, el modelo cognitivo incorpora una función ejecutiva que incluyen "las limitaciones de una memoria de trabajo y procesos de representación que se presentan limitados por un procesador central responsable de la atención selectiva, la inhibición y la planeación" (Norman \& Shallice, 1986, en Martínez y Henao, 2006, p.515). Sally Ozonoff, en el año 1995, propuso la teoría del déficit en las funciones ejecutivas de las personas con autismo (Ozonoff, 1997). El concepto en sí, define la actividad de un conjunto de procesos cognitivos vinculados, históricamente, al funcionamiento de los lóbulos frontales del cerebro (Félix, 2005), ello abarca un amplio abanico de operaciones cognitivas, donde las personas con SA

\footnotetext{
${ }^{4}$ Carol Gray cuenta con numerosas publicaciones en inglés para trabajar las historias sociales (http://www.thegraycenter.org/). No obstante, el libro de Garrigós, A. (2010) ofrece una gama de intervenciones útiles en castellano para trabajar las historias sociales a través de pictogramas.
} 
también presentan dificultades en relación a la planificación, flexibilidad del comportamiento y la búsqueda organizada de elementos.

En términos generales, la investigación de las funciones ejecutivas cerebrales ha despertado en los últimos años un creciente interés, siendo abundantes los estudios que abordan dicho dilema. Al mismo tiempo se denota que las funciones ejecutivas guardan relación con un conjunto de estrategias que incluirían: “( a) Intención de inhibir una respuesta o demorarla para un momento posterior más adecuado; (b) un plan estratégico de secuencia de acción; y (c) una representación mental de la tarea que incluya la información de los estímulos relevantes codificada en la memoria y la meta futura deseada, operaciones que sirven para controlar y regular el procesamiento de la información a lo largo del cerebro" (Félix, 2005, p. 218). Las implicaciones de las funciones ejecutivas y los principios adecuados de intervención, según Cumine, Leach y Stevenson (2000), serían los siguientes:

\begin{tabular}{|l|l|}
\hline \multicolumn{2}{|c|}{ DIFICULTADES Y PRINCIPIOS DE INTERVENCIÓN A NIVEL ESCOLAR } \\
\hline \multicolumn{1}{|c|}{ DIFICULTADES EN } & \multicolumn{1}{c|}{ PRINCIPIOS DE INTERVENCIÓN } \\
\hline La percepción de la emoción & $\begin{array}{l}\text { Las tareas a realizar deben estar claramente } \\
\text { explicadas, de preferencia, con pasos } \\
\text { previamente diseñados. }\end{array}$ \\
\hline La imitación & Se recomienda especificar sub-objetivos. \\
\hline En el juego de simulación & $\begin{array}{l}\text { Es recomendable ayudarles a identificar la idea } \\
\text { principal en la nueva información. }\end{array}$ \\
\hline En la planificación & $\begin{array}{l}\text { Favorecerles en la asociación de los } \\
\text { conocimientos nuevos con los previos. }\end{array}$ \\
\hline $\begin{array}{l}\text { Inconvenientes para comenzar y terminar } \\
\text { las tareas }\end{array}$ & $\begin{array}{l}\text { Ayudarles a ver la imagen completa, en vez de } \\
\text { fijarse sólo en los detalles. }\end{array}$ \\
\hline
\end{tabular}

Figura 4. Las implicaciones de las funciones ejecutivas y los principios adecuados de intervención, según Cumine, Leach y Stevenson (2000),

Queremos destacar que, en el momento de planificar la intervención educativa al alumnado con trastorno autista, es de suma importancia tener presente los déficits que se explican a través de estas tres teorías para aplicar los principios e intervención en el centro escolar y en la propia aula ordinaria y, de ese modo, poder responder a sus necesidades educativas especiales (n.e.e.). 


\section{Estrategias para la clase}

Se debe recordar que cada persona con SA es única, por lo tanto, habría que planificar estrategias apropiadas a las necesidades de cada una. Sin embargo, existen una serie de claves que pueden resultar comunes a la hora de intervenir con este tipo de alumnado y son las áreas que a continuación especificamos:

\begin{tabular}{|c|c|}
\hline INTERVENCIONES GENERALES & MODALIDAD DE TRABAJO \\
\hline $\begin{array}{l}\text { Según el Equipo Deletrea (2004), las estrategias } \\
\text { generales de intervención abarcarían los } \\
\text { siguientes aspectos: }\end{array}$ & $\begin{array}{l}\text { El modo y la forma en que se debe proceder en } \\
\text { el aula serían (Cumine, Leach y Stevenson, } \\
\text { 2000): }\end{array}$ \\
\hline $\begin{array}{l}\text { Empleo constante de apoyos visuales en } \\
\text { cualquier proceso de enseñanza. }\end{array}$ & Crear un clima de trabajo tranquilo. \\
\hline $\begin{array}{l}\text { Asegurar un ambiente estable y predecible, } \\
\text { evitando cambios inesperados. }\end{array}$ & $\begin{array}{l}\text { Garantizar que la estructura de la clase sea } \\
\text { clara. }\end{array}$ \\
\hline $\begin{array}{l}\text { Favorecer la generalización de los } \\
\text { aprendizajes. }\end{array}$ & $\begin{array}{l}\text { Asegurarse de que la persona ha entendido lo } \\
\text { que se espera de ella. }\end{array}$ \\
\hline Asegurar pautas de aprendizaje sin errores. & $\begin{array}{l}\text { Clasificar las tareas, aumentando gradualmente } \\
\text { las demandas. }\end{array}$ \\
\hline $\begin{array}{l}\text { Descomponer las tareas en pasos más } \\
\text { pequeños. }\end{array}$ & $\begin{array}{l}\text { Dirigir la atención del estudiante con SA a nivel } \\
\text { individual, en lugar de confiar en las } \\
\text { instrucciones a toda la clase. }\end{array}$ \\
\hline Ofrecer oportunidades de hacer elecciones. & $\begin{array}{l}\text { Registrar y realizar un seguimiento de los } \\
\text { avances. }\end{array}$ \\
\hline $\begin{array}{l}\text { Ayudar a organizar su tiempo libre, evitando la } \\
\text { inactividad o la dedicación excesiva a sus } \\
\text { intereses especiales. }\end{array}$ & Evaluar las estrategias de intervención. \\
\hline $\begin{array}{l}\text { Enseñar de manera explícita habilidades y } \\
\text { competencias que, por lo general, no suelen } \\
\text { requerir una enseñanza formal y estructurada. }\end{array}$ & $\begin{array}{l}\text { Trabajar en colaboración con otros apoyos } \\
\text { (profesionales...) y establecer vínculos entre la } \\
\text { escuela y la familia. }\end{array}$ \\
\hline $\begin{array}{l}\text { Priorizar objetivos relacionados con los rasgos } \\
\text { nucleares del SA. }\end{array}$ & \\
\hline $\begin{array}{l}\text { Incluir temas de interés generales y } \\
\text { particulares. }\end{array}$ & \\
\hline $\begin{array}{l}\text { Prestar atención a los indicadores emocionales. } \\
\text { Evitar en lo posible la crítica y el castigo. }\end{array}$ & \\
\hline
\end{tabular}

Figura 5. Estrategias generales de intervención asumidas 
No obstante, hay que reconocer que los docentes de apoyo (profesorado de pedagogía terapéutica, etc.), tienen un rol esencial para aumentar la confianza del resto del profesorado en la educación de los estudiantes con SA. Es evidente que los docentes o el personal de apoyo educativo no siempre tendrán todas las respuestas, de ahí que el trabajo colaborativo de los profesionales sea el apoyo ideal para ir encontrando las respuestas necesarias, no olvidando que aspectos como la rutina, la claridad y la consistencia son claves para la intervención educativa. De igual modo, los pequeños cambios ambientales en el aula pueden ayudar al estudiante con SA en sus dificultades en las áreas del entorno físico; el lenguaje y la comunicación; el entorno social; y el curriculum, aspectos que describiremos a continuación:

Un entorno físico impredecible e improvisado confunde a las personas con SA, por lo tanto, la organización y ámbitos estructurados resultan fundamentales. El programa TEACCH es un programa estatal de Carolina del Norte que se desarrolló a mediados de la década de 1960 con el objetivo de ayudar a las personas con trastorno de espectro autista y a sus familias (Mesibov y Howley, 2010). Se pretende que estas personas tengan una vida en la comunidad a través de una intervención estructurada. Para algunas personas la utilización de este método es considerado como la colocación de andamios y para otras como prótesis.

Autores como Tsang, Shek, Lam, Tang y Cheung (2007), argumentan la eficacia de la metodología de este programa. Por otra parte, entre los principales elementos destacables de TEACCH podemos señalar los siguientes (Cumine, Leach y Stevenson, 2000; Wall, 2007; Pittman, 2007; Taylor y Preece, 2010; Mesibov y Howley, 2010):

- Estructura física: esto hace referencia a la forma en la que se organiza el entorno. Separar entornos visualmente claros para entender lo que se espera en cada área. Por ejemplo, en el área de trabajo las distracciones tienen que ser mínimas.

- Agendas (horarios): ayuda a los estudiantes a entender la actividad que va a llevarse a cabo. Usando objetos, fotos, imágenes, números, palabras (dependiendo del nivel de desarrollo de cada estudiante), la persona con SA podrá entender la secuencia de los eventos. 
- Sistemas de trabajo: a través de estos sistemas se les enseña a saber qué trabajo hay que realizar; cuándo se debe terminar; qué pasará cuando se termine...

- Claridad visual: las tareas se presentan visualmente para resaltar la información más importante y se comprenda lo que hay que hacer.

El utilizar ayudas visuales para la organización es muy señalado por las personas expertas (Jordan y Powell, 1995; Winter, 2005; Betts et. al, 2007; Mesibov y Howley, 2010). Además de estas ayudas se aconseja situar al estudiante con SA cerca de la puerta del aula. Por otra parte, resulta novedoso que algunos de estos protagonistas han plasmado sus vivencias en libros explicando la importancia del apoyo visual en sus vidas, como es el caso de Temple Grandin (1984, en Attwood, 2009:409) quien argumenta lo siguiente:

"Mi mente es por completo visual y para mí resulta sencillo el trabajo espacial como el dibujo. Recuerdo que me enseñé a mí misma a dibujar en seis meses. He diseñado grandes edificios de acero y cemento como establos para alojar ganado, pero me sigue resultando difícil recordar un número de teléfono o sumar”.

El lenguaje y la comunicación. Las personas con SA suelen tener un buen lenguaje, con abundante vocabulario, estructuras gramaticales correctas, etc. Sin embargo, tienen dificultades en la comunicación sobre todo en la pragmática (uso del lenguaje) y en la semántica. Por lo tanto, uno de los objetivos es el ayudar a estas personas a desarrollar la intención comunicativa; desarrollar su capacidad para iniciar o mantener una conversación; mejorar la comprensión. Las siguientes estrategias pueden resultar útiles:

\begin{tabular}{|l|l|}
\hline La estructuración del entorno lingüístico & Desarrollo de la comprensión \\
\hline $\begin{array}{l}\text { Cuando se vaya a dar alguna instrucción a } \\
\text { toda la clase, decir su nombre. }\end{array}$ & $\begin{array}{l}\text { Tener algún intérprete que pueda ayudar al } \\
\text { estudiante con SA a entender el sentido de } \\
\text { las palabras. }\end{array}$ \\
\hline $\begin{array}{l}\text { Fomentar y reforzar todos los intentos para } \\
\text { comunicarse. }\end{array}$ & $\begin{array}{l}\text { Si el estudiante con SA tiene intereses } \\
\text { concretos, "usarlos" para el trabajo. }\end{array}$ \\
\hline $\begin{array}{l}\text { Uso concreto, directo y explícito de las } \\
\text { instrucciones, apoyado con imágenes. }\end{array}$ & $\begin{array}{l}\text { Ayudar al estudiante con SA a variar el tono } \\
\text { de voz dependiendo de la situación. }\end{array}$ \\
\hline $\begin{array}{l}\text { Dar tiempo para que respondan y } \\
\text { posteriormente comprobar que han entendido. }\end{array}$ & Fomentar el contacto visual. \\
\hline
\end{tabular}




\begin{tabular}{|l|l|}
\hline Si es necesario repetir la instrucción. & $\begin{array}{l}\text { Enseñarles algunas metáforas y explicarles } \\
\text { el significado. }\end{array}$ \\
\hline $\begin{array}{l}\text { Enseñarles alguna frase hecha para que la } \\
\text { puedan usar cuando no entiendan el } \\
\text { significado de algo. }\end{array}$ & $\begin{array}{l}\text { Ayudarles a entender el significado y las } \\
\text { emociones después de las expresiones } \\
\text { faciales. Llamar la atención sobre } \\
\text { expresiones faciales en libros, espejos, etc. }\end{array}$ \\
\hline Evitar la ironía y los sarcasmos.
\end{tabular}

Figura 6. Estrategias para la comunicación lingüística

El entorno social. No debemos forzar a los estudiantes con SA a jugar, pero sí les debemos enseñar estrategias de cómo hacerlo. Se debe tener en cuenta la ansiedad que suelen sentir estos estudiantes al realizar trabajos en grupo. Se pueden ofrecer oportunidades para que estos escolares, en ocasiones, tengan su espacio. Por lo general, suelen preferir estar con personas mayores antes que con las personas de su edad, debido a que los adultos se adaptan mejor a sus necesidades. También hay que tener presente que un porcentaje elevado de escolares con SA son víctimas del bullying (Dubin, 2007; Attwood, 2009). Los ejemplos dados por estos autores nos ofrecen pistas de cómo deben planificarse algunas estrategias adecuadas adaptadas a las necesidades de estos estudiantes.

Sabemos que el bullying implica un desequilibrio de poder, lo que deriva a utilizar la agresión y las personas con SA son susceptibles de ser víctimas de esta acción agresiva y maltratadora. Si se revela el diagnóstico en la clase habrá que remarcar aspectos positivos y utilizar ejemplos de conducta ejemplar que tengan. Tampoco se debe olvidar que resulta necesario mantener una comunicación fluida con la familia para una colaboración conjunta.

En base a estas apreciaciones, nuestra intervención debería abordar los siguientes aspectos (Cumine, Leach y Stevenson, 2000; Winter, 2005; Attwood, 2009):

- Garantizar que todo el personal de la escuela conozca las dificultades sociales que muestran estas personas y preparar una intervención adecuada trabajando en equipo.

- Con el consentimiento de la familia, puede ser útil hablar en la escuela y en la clase sobre el SA.

- Trabajar en la escuela para prevenir el bullying.

- Enseñarle cómo responder a comportamientos no deseados.

- Asegurarse de que sabe a qué adulto acudir cuando se sienta mal. 
- Contar con una zona tranquila en la que se pueda retirar cuando se sienta ansioso. En esta zona, que podría ser alguna esquina de la clase, se deberían consensuar unas normas para poder pasar un tiempo ahí.

- Analizar el grupo clase y elegir a los compañeros y compañeras que puedan formar "tutoría entre iguales" o un "círculo de amigos". La modalidad de "círculo de amigos" es un mecanismo de ayuda social durante las tareas de grupo, tiempos de recreo y de otras actividades, tanto lúdicas como formales. Generalmente lo forman seis u ocho personas voluntarias quienes se reúnen regularmente con la persona del foco de atención y un adulto. El círculo, según Plimley y Bowen (2007), tiene tres funciones principales: ofrecer ánimos y reconocimiento de éxitos; identificar dificultades y elaborar estrategias para alcanzar objetivos; ayudar para poner estas ideas en la práctica. Por lo tanto, los pares de iguales es un recurso natural que puede ayudar en la intervención con los estudiantes con SA explicándoles por qué su tarea es tan importante y necesaria.

- Utilizar historias sociales por ejemplo las de Carol Gray (como ya se ha comentado anteriormente), para ayudar a las personas con SA a entender las situaciones sociales.

El entorno curricular. La mayoría de estos escolares estudian en aulas ordinarias, por lo tanto el profesorado necesita mantenerse informado, ser tolerante, empático y estar conscientes de que todo el centro debe sentirse implicado en responder a las necesidades del alumnado con estas características.

Por lo general, el estilo de aprendizaje de los estudiantes con SA está compuesto por las siguientes características (Cumine, Leach y Stevenson, 2000):

- Motivación: no suelen ser nada competitivos y no suelen sentir ese deseo de destacar.

- Imitación: les suele resultar difícil imitar los movimientos de los demás ajustándolos a su marco de referencia.

- Percepción: existe la posibilidad de respuestas incoherentes o inesperadas a la entrada sensorial.

- Atención: el foco de interés suele ser a menudo obsesivo.

- Memoria: la memoria suele ser episódica, por ejemplo, los eventos no suelen ser contados en su contexto. Pueden recoger una lista interminable de datos sin relacionarlos entre sí. 
- Secuenciación: muestran dificultades con las secuencias ya que pueden llegar a copiar el modelo, pero les resulta difícil obtener el principio en que se basa.

- Resolución de problemas: pueden aprender una variedad de estrategias, pero no ser conscientes de que las poseen y por lo tanto no seleccionar la estrategia apropiada para una nueva situación.

A modo de ejemplo, incluimos las siguientes dificultades y estrategias a seguir:

\begin{tabular}{|l|l|}
\hline \multicolumn{1}{|c|}{ DIFICULTAD } & \multicolumn{1}{c|}{ ESTRATEGIA } \\
\hline \multicolumn{2}{|c|}{ En el área de matemáticas } \\
\hline $\begin{array}{l}\text { Dificultad para entender las } \\
\text { instrucciones completas. }\end{array}$ & $\begin{array}{l}\text { Simplifique su lenguaje. Siga instrucciones paso } \\
\text { a paso en el tiempo. Use objetos e imágenes } \\
\text { como soporte para que entienda. }\end{array}$ \\
\hline $\begin{array}{l}\text { El estudiante está fascinado con los } \\
\text { números y pregunta una y otra vez } \\
\text { interrumpiendo la lección. }\end{array}$ & $\begin{array}{l}\text { Establecer una norma clara. Decirle por ejemplo } \\
\text { que sólo puede realizar la misma pregunta tres } \\
\text { veces e intentar dedicar tiempo para aclararle } \\
\text { sus dudas. }\end{array}$ \\
\hline $\begin{array}{l}\text { Presentan dificultades para entender el } \\
\text { lenguaje de las matemáticas. }\end{array}$ & $\begin{array}{l}\text { Usar ejemplos prácticos para ayudarle a } \\
\text { entender los ejercicios. }\end{array}$ \\
\hline $\begin{array}{l}\text { No está seguro de cómo responder a la } \\
\text { pregunta ¿por qué? }\end{array}$ & $\begin{array}{l}\text { Cuando sea posible, convierte las preguntas en } \\
\text { afirmaciones dejando un hueco para que el } \\
\text { estudiante pueda responder. }\end{array}$ \\
\hline \multicolumn{1}{|c|}{ En el área de ciencias } \\
\hline $\begin{array}{l}\text { El estudiante prefiere trabajar solo y se } \\
\text { resiste a compartir las tareas con los } \\
\text { demás. A la hora del trabajo grupal } \\
\text { suele presentar muchas dificultades. }\end{array}$ & $\begin{array}{l}\text { Tener cuidado en la elección de los miembros } \\
\text { del grupo. Al inicio puede que el estudiante con } \\
\text { SA tenga un papel más pasivo como recoger los } \\
\text { resultados. Se recomienda que de forma gradual } \\
\text { se le vaya otorgando un papel más activo dentro } \\
\text { del grupo. }\end{array}$ \\
\hline $\begin{array}{l}\text { No es capaz de pedir ayuda en las } \\
\text { clases. }\end{array}$ & $\begin{array}{l}\text { Enseñarle a reconocer cuando se queda atascado } \\
\text { e indicarle cómo pedir ayuda cuando la necesite. }\end{array}$ \\
\hline
\end{tabular}

\section{Figura 6. Estrategias curriculares específicas}

Para muchos estudiantes con SA el curriculum no es un problema. Sin embargo, en las áreas no curriculares es en donde más se manifiestan sus dificultades, sobre todo por la falta de estructuración, como por ejemplo en las asambleas, recreos, etc. La siguiente información pretende ofrecer estrategias de buena práctica ante estas situaciones (Cumine, Leach y Stevenson, 2000; Betts et. al, 2007):

- Asambleas: poco a poco se puede incorporar a este tipo de actividades. Como acción previa debe explicársele con claridad las reglas de la asamblea, usando ayudas visuales 
para reforzar que entiende. Permitir que se sienta en el borde de un grupo en vez de en el medio y alentar a algún compañero o compañera a que adopte el rol de ayudante ofreciéndole orientación.

- Recreos: los recreos, al no tener una estructura concreta y ser espacios con mucho ruido, les resultan confusos y tienden a sentirse ansiosos. Sin embargo, estas áreas presentan oportunidades para desarrollar competencias sociales. Por ejemplo, debe crearse un espacio en el que se fomente la colaboración; aceptar que elijan estar por su cuenta; organizar juegos simples, estructurados y sociales donde el rol de cada persona sea claro; animarles a que observen las actividades que se llevan a cabo en el recreo; encontrar alguna actividad que les atraiga y con la ayuda de los pares animarles para que participen; enseñarles temas de conversación útiles que les pueden permitir iniciar y mantener conversaciones.

- Moverse en la escuela: el desplazarse por la escuela de aula a aula, etc. suele resultar estresante para los estudiantes con SA. Las estrategias que se pueden llevar a cabo son: planificar la entrada y salida para que no sea un caos; antes de ir a una escuela por ejemplo la de secundaria, realizar visitas, con antelación, para que puedan aprenderse el recorrido; utilizar los pares para ayudarles.

- En el comedor: este es otro espacio con mucho ruido y bullicio en el que las personas con SA también suelen mostrarse ansiosas. Las estrategias al respecto pueden ser: establecer normas claras, reforzadas con ayudas visuales; considerar que se sienten en una zona tranquila por ejemplo en alguna esquina y no en el medio donde suele haber más alboroto; alertar a los responsables del comedor de las dificultades y de las estrategias a llevar a cabo; enseñarles a pedir ayuda a otras personas cuando lo necesiten; fomentar que los iguales sean ayudantes.

- Días especiales (fiestas, salidas, etc.): en algunas ocasiones la rutina habitual de la escuela suele variar algunos días como por ejemplo en la celebración de algún evento especial. El profesorado debe prevenir a los estudiantes con SA qué es lo que va a ocurrir en esos días, sobre todo para que se vayan anticipando al cambio de rutina. Se 
requiere contar con el programa unos días antes para poder anticipar en qué tipo de actividad podrá participar el estudiante.

- Pedir ayuda y la resolución de problemas: la clave consiste en tener conciencia de cuáles son las tareas en las que muestran más dificultad. Si están teniendo dificultad en algún ejercicio, tratar de trabajar conjuntamente intentando explicarles cuál es la tarea paso a paso; cuando terminen la actividad tratar de hacerles reflexionar sobre su propio aprendizaje; enseñarles a reconocer cuando se atascan y cómo pedir ayuda.

- Trabajando con otras personas: las prácticas para ayudar a los estudiantes con SA a trabajar con los demás radican en conocer cuál es el nivel social que toleran sin sentirse ansiosos; permitir que se sientan en el borde de un grupo en vez de en la mitad, considerando la distribución de los asientos. El estudiante con SA se puede sentir más cómodo si la otra persona se siente en diagonal a él y no a su lado; se pueden utilizar sus intereses para compartir con el grupo, definiendo con claridad la tarea de cada persona dentro del grupo.

Fundamentándonos en las observación y generalizaciones planteadas por Alberta Learning (2003), hemos tratado de aunar esas orientaciones con la de diversos autores y autoras en un todo coherente y facilitador de dato consultivo (Frith, 1989; Wing y Everard, 1989; Asperger, 1991; William, 1995; Happé, 1991; Munro, 1999; Attwood, 2000, 2009; Rivière, 2001; Tortosa y González, 2001; Moreno y O’Neal, 2003; Sepúlveda, 2008), presentando las áreas más complejas que precisan ser consideradas y asumidas en el contexto escolar. Estos criterios recaen en las siguientes categorías: lenguaje, interacción social, insistencia en la invarianza del ambiente, intereses restringidos, escasa habilidad de organización, coordinación motora y concentración, dificultades académicas, vulnerabilidad emocional y exceso de sensibilidad sensorial. En definitiva, se deben conocer las peculiaridades del estudiante con SA y adaptar el entorno de la escuela para facilitar su aprendizaje, contando siempre con el apoyo y colaboración de la familia y otros profesionales 


\section{A modo de síntesis y conclusión ${ }^{5}$}

Realmente, los “actores" de la inclusión lo conforman toda la comunidad educativa: alumnado del centro, profesorado y personal adjunto, así como las familias. Bien es cierto que estas últimas deben beneficiarse de una serie de consideraciones centradas en : una información adecuada, ayuda profesional y apoyo firme, continuado y de permanencia, no lo es menos para el profesorado de aula ordinaria, también éstos requieren de soportes básicos, tales como: formación idónea para aumentar su competencia profesional, servicios de apoyo, espacios de autoformación, análisis, orientación y reflexión con otros profesionales que le permitan intercambiar experiencias y buscar soluciones comunes en situaciones de inclusión, específicamente, con la problemática puntual planteada por el alumnado con SA.

Conviene remarcar además, que en algunos aspectos "el trabajo aislado del profesor permite un cierto grado de seguridad y protección", pero la toma de decisiones discrecionales sobre el alumnado con n.e.e. hace que "se aparte de ese aislamiento y la incertidumbre creada se comparta y discuta" (Fullan, 1994, p.149), para que de ese modo, pueda acceder a un tipo de información compartida y valiosa con el fin de lograr mayor eficacia en la tarea que se está realizando.

Es necesario pensar en un proyecto inclusivo de intervención con aspectos psicopedagógicos, orientado a facilitar al estudiante con SA el acceso a los aprendizajes en las mejores condiciones. Este asesoramiento psicopedagógico resulta válido para optimizar el acto educativo, además, unido a ello, es preciso tener como consideración previa, ciertos factores que debe reunir el centro para que la recepción escolar resulte motivadora (Paula, 2003; Sepúlveda, 2008, 2011):

- Los centros escolares, preferiblemente, deben ser de pequeño tamaño y número bajo de estudiantes por aula.

- No deben existir interacciones de excesiva complejidad, debiendo ser evitados aquellos centros excesivamente bulliciosos y "despersonalizados".

- Son preferibles centros estructurados, con estilos didácticos directivos y formas de organización que hagan "anticipable” la jornada escolar.

\footnotetext{
${ }^{5}$ La síntesis original en la cual se basan algunos fundamentos de estas conclusiones pueden encontrarse en la Revista REPSI. No 113/114. Enero/Abril, pp. 28-40. Santiago de Chile.
} 
- Resulta imprescindible el compromiso real del claustro de profesorado y de los docentes concretos que atienden en sus aulas a los estudiantes con SA.

- De igual modo, resulta trascendental proporcionar a los iguales, claves para comprenderle y apoyar sus aprendizajes y relaciones.

- El proyecto educativo, en general, no debe estar basado en la restrictividad, ya que ello obstaculiza las interacciones de sus protagonistas más esenciales, lo cual dificulta la efectividad en la consecución de sus metas.

Insistimos en señalar la inexistencia de una receta exclusiva que pueda ser aplicada en las aulas para todos los estudiantes con este tipo de trastorno. De la misma manera que, ningún método educativo responde a la necesidad de "todos" y de "cada uno" de los estudiantes que no están afectados por este síntoma, igual ocurre con el alumnado que presenta estas características.

Con lo que aquí se ha descrito, concluimos que en base a las dificultades que el síndrome en sí plantea, se ha intentado principalmente, abordar las concepciones desde la dimensión afectiva, de relación y cognición, sugiriendo la idea de que un mejor conocimiento del tema, posibilita una mejor comprensión e intervención educativa hacia personas con SA. En general, ello permitirá contribuir a un mejor desarrollo de habilidades sociales, de comunicación, de lenguaje, de autorregulación y de autodeterminación, lo que equivale, en síntesis, a potenciar una mejor calidad de vida para estas personas desde el referente educativo en el marco de los centros y aulas inclusivas.

\section{Referencias}

American Psychiatric Association. (1993). Manual Diagnóstico y Estadístico de los trastornos Mentales. DSM-IV. Barcelona: MASSON.

American Psychiatric Association. (2002). Manual Diagnóstico y Estadístico de los trastornos Mentales. DSM-IV-TR. Barcelona: MASSON.

Alberta Learning (2003). Teaching Students with Autism Spectrum Disorders. Edmonton. [ref. de 14 de diciembre de 2010] Disponible en: http://education.alberta.ca/media/511995/autism.pdf 
Asperger, H. (1944). Die autistïchen Psychopathen im Kindesalter. Archiv für Psychiatrie und Nervenkrankenheiten, 117, 76-136.

Attwood, T. (2000). ¿Quizás los niños con un Desorden del Espectro Autista deben ser eximidos de hacer deberes? [ref. de 6 de noviembre de 2004]. Disponible en Web: 〈http://es.geocities.com/sindromedeasperger/Informa/articulos/96.htm>

Attwood, T. (2009). Guía del Síndrome de Asperger. Barcelona: Paidós.

Baron-Cohen, S. (2000). Theory of mind and autism: A fifteen year review. In S. BaronCohen, H. Tager-Flusberg, \& D. J. Cohen (Eds.), Understanding other minds. Perspectives from developmental cognitive neuroscience (pp. 3-21). Oxford: University Press.

Baron-Cohen, S.; Leslie, A.M. y Frith, U. (1985). Does the autistic child have a "theory of mind"? Cognition, 21, 37-46.

Betts, S.W; Betts, D.E. and Gerber-Eckard, L.N. (2007). Asperger Syndrome in the Inclusive Classroom: Advice and Strategies for Teachers. London: Jessica Kingsley Publishers.

Bogdashina, O. (2005). Percepción Sensorial en el autismo y Síndrome de Asperger: Experiencias Sensoriales Diferentes, Mundos Perceptivos Diferentes. Autismo Ávila.

Cumine, V; Leach, J. and Stevenson, G. (2000). Asperger Syndrome: A Practical Guide for Teachers. London: David Fulton Publishers.

Dubin, N. (2007). Asperger Syndrome and Bullying: Strategies and Solutions. London: Jessica Kingsley Publishers.

Equipo Deletrea. (2004). Un acercamiento al Síndrome de Asperger: Una guía teórica y práctica. Valencia: Asociación Asperger España.

Félix, V. (2005). Perspectivas recientes en la Evaluación Neuropsicológica y Comportamental del trastorno del Déficit de Atención con/sin Hiperactividad. Electronic Journal of Research in Educational Psychology, 7 (3)3, 215-232.

Frith, U. (1989). Autism: Explaining the Enigma. Oxford: Blackwell.

Fullan, M. (1994). La gestión basada en el centro; el olvido de lo fundamental. Revista de Educación, 304, 147-61.

Garrigós, A. (2010). Pictogramas y pautas desarrolladas para Síndrome de Asperger: Manual práctico para familiares y profesionales. Valencia: Promolibro. 
Ghaziuddin, M. (2010). Brief Report: Should the DSM V Drop Asperger Syndrome? Journal of Autism and Developmental Disorders. 40 (9), 1146-1148.

Happé, F. (1998). Introducción al Autismo. Madrid: Alianza Editorial.

Jordan, R. and Powell, S. (1995). Understanding and Teaching Children with Autism. England: John Wiley \& Sons.

Klin, A. y Volkmar, M. D. (1996). Síndrome de Asperger. Tratamiento e intervención. Algunas recomendaciones para los padres. Yale: Learning Disabilities Association of America.

Leslie, A.M. (1987). Pretence and representation: The origins of "Theory of mind". Psychological Review, 94, 412-426.

Martínez, M. y Henao, G. (2006). Desempeño en la función ejecutiva de niños expuestos a ambientes de enseñanza bilingüe. Electronic Journal of Research in Educational Psychology, 10 (4)3, 513-528.

Mesibov, G. y Howley. M. (2010). El acceso al currículo por alumnos con Trastornos del Espectro del Autismo: Uso del Programa TEACCH para favorecer la inclusión. Ávila: Autismo Ávila.

Moreno, S. y O’Neal, C. (2003). Consejos para enseñar a personas autistas de alto funcionamiento. [ref. de 15 de noviembre de 2004]. Disponible en Web: <http:// geocitides.com/sindromedeasperger/Informa/articulos/69.htm>

Munro, N. (1999). ¿Cuál es la diferencia entre autismo de alto funcionamiento y el síndrome de Asperger? [ref. de 15 de noviembre de 2003]. Disponible en Web: $\langle$ http://www.autism.com/scripts/ articulo/smuestra.idc?n=munro1>

Myles, B.S; Hagen, K; Holverstott, J; Hubbard, A; Adreon, D. and Trautman, M. (2005). Life Journey through Autism: An Educator's Guide to Asperger Syndrome. Arlington: Organization for Autism Research.

Organización Mundial de la Salud. CIE 10 o ICD-10 (Décima revisión de la Clasificación Internacional de las Enfermedades). Trastornos mentales y del comportamiento. (1992). Zaragoza: Meditor. 
Ozonoff, S. (1997). Components of executive function in autism and other disorders. In Russell (Ed.), Autism as an executive disorder (pp.179-211). Nueva York: Oxford University Press.

Paula Pérez, I. (2003). Educación Especial. Técnicas de Intervención. Madrid: McGraw-Hill /Interamericana de España.

Pittman, M. (2007). Helping Pupils with Autistic Spectrum Disorders to Learn. London: Paul Chapman Publishing.

Plimley, L. Bowen, M. (2007). Social Skills and Autistic Spectrum Disorders. London: Paul Chapman Publishing.

Premack, D., \& Woodruff, G. (1978). Does the chimpanzee have a theory of mind? Behavioral and Brain Sciences, 1(4), 515-526.

Rivière, A. (2001). Autismo: Orientaciones para la intervención educativa. Madrid: Trotta.

Shaffer, R. (2000). Desarrollo Social. México: Siglo XXI editores, S.A. de C.V.

Sepúlveda Velásquez, L. (2009). El síndrome Asperger desde la noción global de autismo: Orientaciones para el aula regular. En C. Medrano y L. Sepúlveda (Coords.) Diferentes perspectivas de atención a la diversidad. Siete enfoques pertinentes a la integración escolar (pp. 135-156). Santiago de Chile: Edt. LOM.

Sepúlveda Velásquez, L. (2008). Actitud docente hacia la integración educativa de alumnos con síndrome autista Asperger o de alto funcionamiento en el aula regular. Tesis doctoral, Universidad de del País Vasco, Bilbao, España.

Sepúlveda Velásquez, L. (2011). Guía orientativa para el profesorado del aula regular relativa a la integración del alumnado con síndrome Asperger. Revista REPSI, 113/114, $28-40$.

Stichter, J.P.; Herzog. M.J.; Visovsky, K.; Schmidt, C.; Randolph, J.; Schultz, T. y Gage, N. (2010). Social Competence Intervention for Youth with Asperger Syndrome and HighFunctioning Autism: An Initial Investigation. Journal of Autism and Developmental Disorders, 40(9), 1067-1079.

Taylor, K. and Preece, D. (2010). Using Aspects of the TEACCH Structured Teaching Approach with Students with Multiple Disabilities and Visual Impairment: Reflections on Practice. British Journal of Visual Impairment, 28(3), 244-259. 
Tsang, S., Shek, D., Lam, L., Tang, F., Cheung, P. (2007). Brief Report: Application of the TEACCH Program on Chinese Pre-School Children with Autism - Does Culture Make a Difference? Journal of Autism and Developmental Disorders, 37(2), 390-396.

Tortosa, F y González, A. (2001). Factores que favorecen la integración en centros educativos de personas con necesidades educativas especiales asociadas a Trastornos Generalizados del Desarrollo. [ref. de 6 de noviembre de 2004]. Disponible en Web: 〈http://es.geocities.com/sindromedeasperger/Informa/articulos/12.htm>

Wall, K. (2007). Education and care of adolescents and adults with autism: A guide for professionals and carers. London: SAGE Publications.

Williams, K. (1995). Comprender al estudiante con Síndrome de Asperger. Orientación para profesores. [ref. de 6 de noviembre de 2004]. Disponible en Web: <http://es.geocities.com/sindromedeasperger/Informa/articulos/68.htm $>$

Winter, M. (2005). Asperger Syndrome. What Teachers Need to Know. London: Jessica Kingsley Publishers.

Wing, L., Everard, M. P. y otros. (1989). Autismo infantil. Aspectos médicos y educativos. Madrid: Santillana. Aula XXI. 